\title{
Review
}

Frédéric Grillot*, Justin C. Norman, Jianan Duan, Zeyu Zhang, Bozhang Dong, Heming Huang, Weng W. Chow and John E. Bowers

\section{Physics and applications of quantum dot lasers for silicon photonics}

https://doi.org/10.1515/nanoph-2019-0570

Received December 31, 2019; accepted April 14, 2020

\begin{abstract}
Photonic integrated circuits (PICs) have enabled numerous high performance, energy efficient, and compact technologies for optical communications, sensing, and metrology. One of the biggest challenges in scaling PICs comes from the parasitic reflections that feed light back into the laser source. These reflections increase noise and may cause laser destabilization. To avoid parasitic reflections, expensive and bulky optical isolators have been placed between the laser and the rest of the PIC leading to large increases in device footprint for on-chip integration schemes and significant increases in packaging complexity and cost for lasers co-packaged with passive PICs. This review article reports new findings on epitaxial quantum dot lasers on silicon and studies both theoretically and experimentally the connection between the material properties and the ultra-low reflection sensitivity that is achieved. Our results show that such quantum dot lasers on silicon exhibit much lower linewidth enhancement factors than any quantum well lasers. Together with the large damping factor, we show that the quantum dot gain medium is fundamentally dependent on dot uniformity, but through careful
\end{abstract}

[Correction added after online publication 06 June 2020: ' $C_{r}$ ' in the fifth line of the list (1) under the seciton 4.2 and in the sixth line under the section 4.3 was changed to ' $C$ ']

*Corresponding author: Frédéric Grillot, LTCI, Télécom Paris, Institut Polytechnique de Paris, 19 place Marguerite Perey, 91120, Palaiseau, France; and Center for High Technology Materials, University of New Mexico, Albuquerque, 87106, NM, USA, E-mail: grillot@telecomparis.fr. https://orcid.org/0000-0001-8236-098X

Justin C. Norman, Zeyu Zhang and John E. Bowers: Materials and Electrical and Computer Engineering Departments, University of California, Santa Barbara, California, 93106, USA

Jianan Duan, Bozhang Dong and Heming Huang: LTCI, Télécom Paris, Institut Polytechnique de Paris, 19 place Marguerite Perey, 91120, Palaiseau, France

Weng W. Chow: Sandia National Laboratories, Albuquerque, 87185, NM, USA optimization, even epitaxial lasers on silicon can operate without an optical isolator, which is of paramount importance for the future high-speed silicon photonic systems.

Keywords: dynamical instabilities; nanostructures; quantum dots; semiconductor lasers; silicon photonics.

\section{Introduction}

Silicon photonics is considered a mainstream data-transmission solution for next-generation data centers, compact technologies for high bandwidth density interconnects, high-performance computers, and many emerging applications such as sensors, and light detection and ranging (LIDAR) systems for self-driving vehicles [1, 2, 3]. One of the biggest challenges in scaling photonic integrated circuits (PICs) comes from the parasitic reflections that feed light back into the laser source increasing noise and, ultimately, causing strong laser destabilization [4]. It is well-established that the inefficiency of light emission in silicon requires the integration of a III/V laser chip or optical gain materials onto a silicon substrate through wafer-bonding or flip-chip bonding techniques [5, 6]. However, prior works also showed that hybrid semiconductor lasers heterogeneously integrated onto silicon are quite sensitive to unintentional reflections due to the various active and passive transitions and regrowth interfaces [7]. External optical feedback (EOF) is known to have a major impact on the laser performance [8]. Among the large variety of potential effects, whose presence depends on the external cavity length and EOF strength, coherence collapse (CC) operation is perhaps the most influential and penalizing factor affecting the stability and purity of the laser device [9] as well as the power penalty under modulation [10]. In PICs, EOF effects can be much more detrimental because PICs rely on complex designs where light emitters are tightly assembled with other optical components (e. g., modulators, waveguides, etc.,) to achieve the desired functionality, resulting in several possible optical reflections from the silicon integrated devices. To overcome this problem, expensive and bulky optical isolators have 
been included between the laser and the rest of the PICs, leading to large increases in device footprint for on-chip integration schemes and significant increases in packaging complexity for lasers co-packaged with passive PICs. Without this device, the integrated lasers cannot be protected from reflections. Integrated isolators are usually classified into three categories: those based on non-linear effects; those based on spatiotemporal modulation, and those based on magneto optic effects [11, 12]. The latter is schematically explained in Figure 1 where the polarization of the laser light is converted from transverse electric (TE) to transverse magnetic (TM) after the light experiences a roundtrip through a $45^{\circ}$ Faraday rotator. As the waveguide of the laser cavity is made for TE mode emission, light cannot be recoupled into the active area. Despite much recent progress, the chip-scale integration of such optical isolators having both low-loss and sufficient isolation ratio into photonic circuits is not yet available. [13, 14].

To this end, the development of feedback insensitive lasers is of mainstream interest to alleviate the risk of undesired reflections. A pioneering study reported an InAs/ GaAs quantum dot (QD) transmitter integrated on silicon operating without an optical isolator for core I/O applications, demonstrating significant advances in the field [15]. Other recent accomplishments include but are not limited to semiconductor lasers with an intracavity optical isolator [12], photonic crystal-based Fano nanolasers with CC suppression [16], highly coherent lasers with large quality factors [17] [4], parity-time symmetry distributed feedback (DFB) lasers [18], and tunable hybrid lasers based on III-V reflective semiconductor optical amplifiers [19], among many others. However, despite these achievements demonstrated to date, a light source with absolute feedback insensitivity has yet to be reported, particularly at the system level. In this work, we provide novel insights on epitaxial Fabry-Perot (FP) QD lasers on silicon [20, 21, 22]. Semiconductor lasers with QDs have been especially of great interest with their low bias currents and their apparent

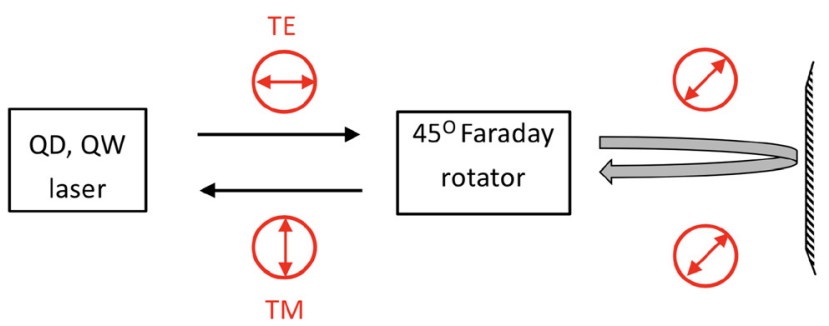

Figure 1: Schematic representation illustrating the principle of the optical isolator with a $45^{\circ}$ Faraday rotator. Due to the reflection on the mirror, the polarization of the laser light is TE-TM converted. QD: quantum dot; QW: quantum well. temperature insensitivity, resulting from the highly confined carriers in the atomic-like electronic structure [23]. Ideally, QDs can be seen as semiconductor atoms with an atom-like density of states leading to an ultimate carrier confinement and enhanced device performances [24-26]. To this end, QD lasers also feature emission linewidths of a few hundreds of $\mathrm{kHz}$ at room temperature [27]. To date, epitaxial QD lasers have demonstrated record performance with threshold currents not exceeding a few milliamps, continuous wave operation up to $105^{\circ} \mathrm{C}$, a very long device lifetime [1], and a small linewidth enhancement factor (i. e., $\alpha_{H}$-factor) [13] along with a high-yield and a much better scalability [28]. From a dynamical viewpoint, QD lasers are very peculiar with strong damping effects to some extent similar to class A lasers [29, 30], while exhibiting low-frequency fluctuations [31] under EOF and anticorrelated fluctuations under polarization rotated feedback [32]. QD lasers also show type II-based excitability featuring strong opto-thermal coupling and birth of square waves [33]. Finally, QD distributed feedback lasers (DFB) demonstrated stronger robustness against parasitic reflections with critical feedback level for CC operation well improved by comparison with quantum well (QW) DFBs [34, 35]. The relative intensity noise (RIN) was also found to be much lower than that of their QW counterparts with the same output power [36]. Finally, under EOF, it was also proved that QD lasers emitting on the sole ground state (GS) transition are by essence much more stable; whereas, those operating on the excited state (ES) or within the dual-state lasing regime (GS + ES) output various complex chaotic dynamical states $[37,38]$. Together, these features strongly contribute to make QD lasers meaningful for many applications including but not limited to isolator-free integrated technologies [4], neuromorphic photonic based systems, optical radars, and high-speed random bit generators [29, 39, 40].

This research article investigates physics and applications of QD lasers for silicon photonics in particular optical feedback instabilities in epitaxial QD lasers. Thus, we show that the defect density can be reduced by means of careful optimization of growth conditions and utilization of dislocation filtering layers and techniques [41]. We discuss the connections between the material properties such as the inhomogeneous broadening due to dot size variations, the gain, the $\alpha_{H}$-factor, and thus, the extremely low reflection sensitivity that can be achieved. To do so, a semiclassical laser model including many-body theory (mbt) is described for gain and linewidth enhancement factor calculations [42]. We show that the latter exhibit values, ranging from negative to positive, which suggests the possibility of a near-zero $\alpha_{H}$-factor at peak gain providing that the inhomogeneous broadening is well-controlled. 
Our experimental results confirm the predictions that QD lasers are capable of achieving much lower $\alpha_{H}$-factors relative to any QW lasers. Apart from the $\alpha_{H}$-factor, other dynamical and nonlinear features, such as the damping factor and the relaxation frequency, are also investigated because of their strong importance for reflection insensitivity performance. Overall, this work provides novel insights for designing high performance reflection insensitive semiconductor lasers, withstanding feedback rates much above the requirements dictated by the IEEE 802.3. These results clearly raise the possibility to integrate epitaxial QD lasers and other optical components without need of an optical isolator.

\section{Quantum dot growth and laser structures}

InAs QDs grow via the kinetically constrained StranskiKrastanov growth mode [26, 43, 44]. In this regime the deposited material initially incorporates as a two-dimensional layer that abruptly transitions to three-dimensional nanostructures upon reaching a critical thickness that is dictated by the growth conditions and strain of the growing layer. Being a self-assembly process, QD growth is highly constrained by atom mobility on the episurface, which is influenced by a host of conditions including: substrate temperature, V/III flux ratio, InAs growth rate, punctuated vs. continuous growth, and the underlying material composition and surface roughness. Equally important to the growth conditions of the dots themselves are the capping and annealing procedures used because In-Ga interdiffusion changes the effective size and homogeneity of the dot layer [45]. The challenge posed by interdiffusion makes molecular beam epitaxy (MBE) a favorable growth technique for QDs relative to metalorganic chemical vapor deposition (MOCVD) due to the lower temperatures required and is the approach taken here. Together, the growth conditions of QDs represent a large and highly coupled parameter space that must be carefully optimized to obtain low inhomogeneous broadening, usually represented by the photoluminescence full-width-at-half-maximum (FWHM). Broad coverage of the effects of different growth conditions on the optical properties of QDs have been presented in [43, 46, 47]. Furthermore, growth conditions that yield narrow inhomogeneous broadening do not necessarily yield high dot density for high gain, so both parameters must be considered during optimization. To obtain a narrow inhomogeneous broadening of lower than $30 \mathrm{meV}$ requires extensive optimization and iteration on each growth condition, and a detailed presentation is beyond the scope of this article [20]. In brief, growth temperature is generally found to be the most critical parameter with a tolerance of lower than $5^{\circ} \mathrm{C}$ about the optimum to achieve narrow PL simultaneously with high gain. The optimum temperature is strongly coupled to the V/III ratio during growth, so they must be optimized together. Other commonly adopted conditions are the use of $\mathrm{As}_{4}$ instead of $\mathrm{As}_{2}$, the addition of growth interruptions [48], and an "In-flush" [49] step where dot inhomogeneity is annealed away through optimized capping layer thickness and a short anneal at $580^{\circ} \mathrm{C}$. Following the In-flush, growth temperatures should be kept low to minimize further inter-diffusion, which diminishes, blue-shifts, and broadens the photoluminescence. The devices in this study utilize dot-in-a-well active regions composed of $\mathrm{In}_{.15} \mathrm{Ga}_{.85} \mathrm{As}$ QWs asymmetrically encompassing the InAs dots with a $2 \mathrm{~nm}$ "prelayer" below and $5 \mathrm{~nm}$ "capping layer" on top. The growth conditions of these layers have a strong impact on the emission wavelength and inhomogeneous broadening. Table 1 summarizes the MBE growth conditions used in this study. Representative photoluminescence from a single dot layer grown on a GaAs substrate is shown in Figure 2(a).

The InAs/GaAs FP laser structure is displayed in Figure 2(b). The laser active region consists of QD layers separated by a $37.5 \mathrm{~nm}$ GaAs spacer. In order to improve the thermal stability, all lasers are p-doped with a doping level of $5 \times 10^{17} \mathrm{~cm}^{-3}$ corresponding to 10 holes/dot. Indeed, it is well-known that the thermal spreading of holes is one of the main mechanisms causing the gain to be temperature sensitive in a QD laser. By introducing p-type modulation doping in the GaAs spacer layers between dot layers, the effect of the closely spaced whole energy levels can be countered so that the QDs' GS transition is always filled by holes. The rear facet of the laser cavity is made with a high power reflectivity of $99 \%$ (HR), while that of the front facet

Table 1: Summary of optimized quantum dot (QD) growth conditions for InAs quantum dots-in-a-well grown by molecular beam epitaxy. ML stands for monolayer (hereafter ML).

\begin{tabular}{ll}
\hline Parameter & Optimized Value \\
\hline Substrate Temperature & $495^{\circ} \mathrm{C}$ \\
Quantum Dot V/III & 35 \\
As Species & $\mathrm{As}_{4}$ \\
Total InAs Deposition & $2.55 \mathrm{ML}$ \\
InGaAs Prelayer/Cap Composition & $\ln _{.15} \mathrm{Ga} .85 \mathrm{As}$ \\
InGaAs Prelayer V/III & 10 \\
InGaAs Cap V/III & 35 \\
InGaAs Prelayer Thickness & $2 \mathrm{~nm}$ \\
InGaAs Cap Thickness & $5 \mathrm{~nm}$ \\
\hline
\end{tabular}


(a)

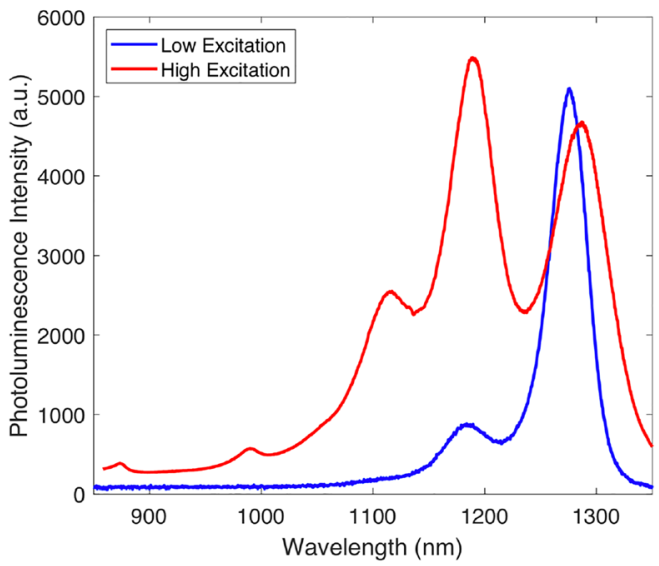

(b)

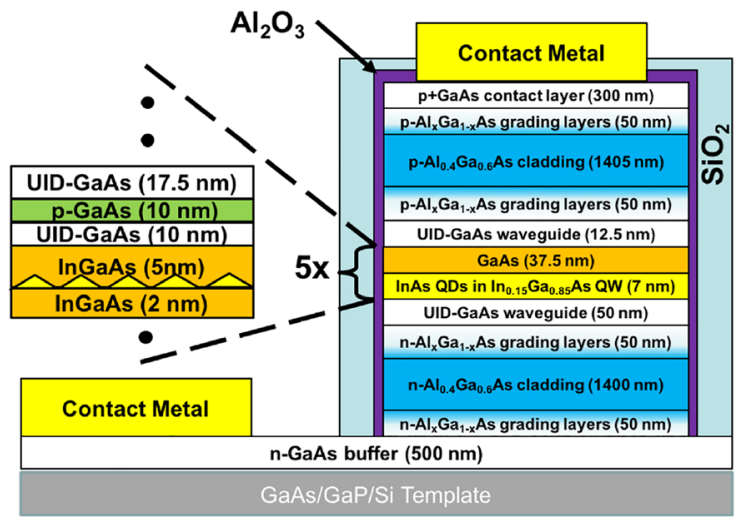

Figure 2: (a) Photoluminescence spectra of an optimized single dot-in-a-well active region at a low pumping level (blue curve) and high pumping level (red curve); (b) Schematic illustration of the p-doped QD laser epitaxial structure. The close-up on the left depicts one period of the active region.

is either left as-cleaved (C) and equals $32 \%$, or coated for a $55 \%$ or $77 \%$ reflectivity. The cavities of the lasers are measured at relatively similar lengths $(L)$, namely $1.35 \mathrm{~mm}$ for the C/HR laser, $1.20 \mathrm{~mm}$ for the 55/HR laser, and $1.55 \mathrm{~mm}$ for the $77 / \mathrm{HR}$ laser. The width of the wide ridge waveguide is fixed at $3 \mu \mathrm{m}$. The increase of the front facet reflectivity is known to reduce the transmission loss $\alpha_{m}$ lowering the threshold current as shown in the light-current $(P-I)$ characteristics depicted in Figure 3(a). The threshold current $I_{S}$ of the QD lasers decreases from $24 \mathrm{~mA}$ (C/HR) to $21 \mathrm{~mA}$ (77/HR) then down to $15 \mathrm{~mA}$ (55/HR). Note that the external efficiency $\left(\eta=\frac{q \lambda}{h c} \frac{\Delta P}{\Delta I}\right.$ with $h$ the Planck constant, $c$ the celerity of light, $q$ the electron charge, and $\lambda$ the lasing wavelength) is expected to increase with the power reflectivity of the output facet. The external efficiency $\eta$ of the QD lasers ranges from $8.5 \%$ (C/HR) to $11.6 \%$ (77/HR) then goes down to $9.8 \%$ (55/HR). Here the $77 / \mathrm{HR}$ laser has a higher threshold and a lower external efficiency than the 55/HR laser which may be attributed to a possible contamination in the coating process and to the HR reflectivity that is not lower than targeted [3].

As shown in Table 2, a decrease of the total loss transforms into a higher quality factor $Q$. Thus, the $Q$ is defined such as $Q=2 \pi v \tau_{p}=2 \pi v /\left[v_{g}\left(\alpha_{i}+\alpha_{m}\right)\right]$ with $v$ the oscillation frequency, $v_{g}$ the group velocity, $\alpha_{i}$ the internal total loss and $\tau_{p}$ the cavity photon lifetime. Let's stress that generally, the optical resonator quality factor is not defined with the mirror loss coefficient but as $Q^{-1}=2 P / 2 \pi \nu W$ where $W$ is the stored energy in the cavity and $P$ is the power escaping one side of the cavity [50,51]. Nevertheless, here, one see that a higher $Q$ mitigates the losses thus increasing the cavity photon lifetime, which is in favor of better laser stability against EOF as discussed hereafter. Figure 3(b) also shows the optical spectra for the three lasers measured (a)

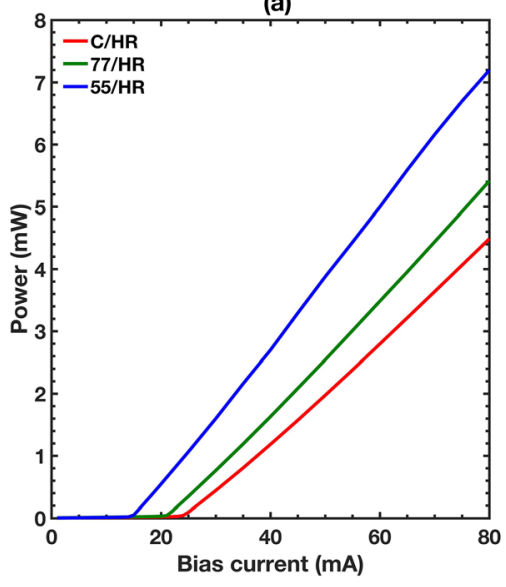

(b)

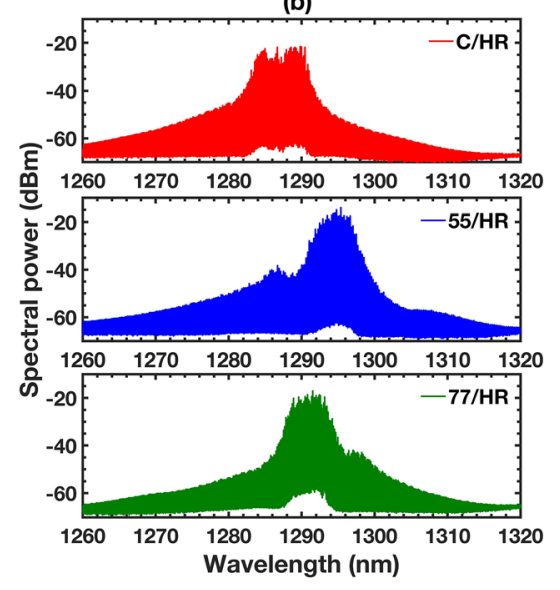

Figure 3: (a) Light-current characteristics of the QD lasers with different facet reflectivity of $\mathrm{C} / \mathrm{HR}, 55 / \mathrm{HR}$ and $77 / \mathrm{HR}$, respectively; (b) Optical spectra measured at $3 \times I_{s}$ for QD lasers with different facet reflectivity of $\mathrm{C} /$ HR, 55/HR and 77/HR, respectively. 
at $3 \times I_{s}$. The lasing emission is shifted from $1285 \mathrm{~nm}(\mathrm{C} / \mathrm{HR})$ to $1290 \mathrm{~nm}$ (77/HR) then to $1295 \mathrm{~nm}$ (55/HR). This red shift of the emission spectrum is usually attributed to wavelength dependence of the gain and the power reflectivity of the mirror coatings. From Figure 3(b), it turns out that the 55/HR case is more red-shifted due to more thermal effects taking place in this laser having the shortest cavity length. All of the epitaxial QD laser on silicon parameters are displayed in Table 2.

\section{Gain and linewidth enhancement factor}

When studying laser linewidth, chirp during high-speed modulation, and optical feedback sensitivity, an important gain-medium parameter to consider is the so called linewidth enhancement factor [52, 53]:

$$
\alpha_{H}=-2 K \frac{d(\delta n)}{d N_{e}}\left(\frac{d G}{d N_{e}}\right)^{-1}
$$

where $\delta n$ is the carrier-induced refractive index, $G$ is the gain, $N_{e}$ is the carrier density and $K$ is the lasing wavevector. This section describes the $\alpha_{H}$-factor in a QD active region, in particular, its behavior at the gain peak. The calculated results are based on a mbt with dephasing effects from carrier scattering treated at the level of quantum-kinetic equations. As shown in (1), the determination of $\alpha_{H}$-factor requires the carrier density dependencies of gain and carrier-induced refractive index. We obtain them by evaluating [54]

$$
\begin{aligned}
{[2 K \delta n(v)+i G(v)] \varepsilon=} & \frac{2 v}{\varepsilon_{0} n_{B} c h_{Q W}}\left[\sum_{n} \wp_{n} \sum_{q} n_{n, q}^{i n h} p_{n, q}(v)\right. \\
& \left.+\frac{1}{A} \sum_{k} \wp_{k} p_{k}(v)\right]
\end{aligned}
$$

where $v$ is the lasing frequency, $c$ and $\varepsilon_{0}$ are the vacuum speed of light and permittivity, $n_{B}$ is the background refractive index, and $h_{Q W}$ and $A$ are the width and area of the QW embedding the QDs. A weak laser probe field $\varepsilon$ is used to extract the susceptibility, which depends on the QD and QW dipole matrix elements, $\wp_{n}$ and $\wp_{k}$, and on $n_{n, q}^{\text {inh }}$, the density of QDs with electronic structure, labeled by $q$, contributing to the $n^{\text {th }} \mathrm{QD}$ transition.

The QD and QW polarizations are obtained from solving the equations of motions,

$$
\begin{aligned}
\frac{d p_{n, q}}{d t}= & i\left(v-\omega_{n, q}^{(0)}\right) p_{n, q}-i \frac{2 \wp_{n} \varepsilon}{\hbar}\left(f_{n, q}^{e}+f_{n, q}^{h}-1\right) \\
& +\frac{i}{\hbar} \sum_{k} V_{n, k}\left(f_{k}^{e}+f_{k}^{h}\right) p_{n, q}+\frac{i}{\hbar} \sum_{k} V_{n, k} p_{k}+S_{n}^{c-p}+S_{n}^{c-c}
\end{aligned}
$$

and,

$$
\begin{aligned}
\frac{d p_{k}}{d t}= & i\left(v-\omega_{k}^{(0)}\right) p_{k}-i \frac{2 \wp_{k} \varepsilon}{\hbar}\left(f_{k}^{e}+f_{k}^{h}-1\right) \\
& +\frac{i}{\hbar}\left[A \sum_{n} V_{n, k} \sum_{q} n_{n, q}^{i n h}\left(f_{n, q}^{e}+f_{n, q}^{h}\right)+\sum_{k^{\prime}} V_{\left|k-k^{\prime}\right|}\left(f_{k^{\prime}}^{e}+f_{k^{\prime}}^{h}\right)\right] p_{k} \\
& +\frac{i}{\hbar}\left[A \sum_{n} V_{n, k} \sum_{q} n_{n, q}^{i n h} p_{n, q}+\sum_{k^{\prime}} V_{\left|k-k^{\prime}\right|} p_{k^{\prime}}\right]+S_{k}^{c-p}+S_{k}^{c-c}
\end{aligned}
$$

In each equation, the first line contains the singleparticle contributions from frequency detuning and stimulated emission, where $\omega_{n, q}^{(0)}$ and $\omega_{k}^{(0)}$ are the unrenormalized transition frequencies, $f_{n, q}^{\sigma}$ and $f_{k}^{\sigma}$ (with $\sigma=e$ (for electrons) or $h$ (for holes) are the QD and QW carrier populations, respectively). The renormalizations of the single-particle contributions are described by the exchange term with Coulomb potential energy matrix element $V_{n, k}$ and the excitonic term with Coulomb potential energy matrix element $V_{\left|k-k^{\prime}\right|}$. The last line of each equation are the higher-order many-body contributions describing dephasing and screening. The full expressions are lengthy and may be found elsewhere $[54,55,56]$. We represent them with $S_{n}^{c-p}$ and $S_{n}^{c-c}$, for carrier-phonon and carrier-carrier scattering, respectively.

For $\alpha_{H}$-factor in a QD active medium, many-body Coulomb effects are important because they cause the QD resonances to frequency shift and broaden with changing

\begin{tabular}{|c|c|c|c|c|c|c|c|}
\hline Laser & $L(\mathrm{~mm})$ & $v(\mathrm{THz})$ & $\eta(\%)$ & $\alpha_{i}\left(\mathrm{~cm}^{-1}\right)$ & $\alpha_{m}\left(\mathbf{c m}^{-1}\right)$ & $\tau_{p}(p s)$ & $Q$ \\
\hline $\mathrm{C} / \mathrm{HR}$ & 1.35 & 233.5 & 8.5 & 34.0 & 4.7 & 3.0 & 4,400 \\
\hline $55 / \mathrm{HR}$ & 1.20 & 231.7 & 11.6 & 15.6 & 3.1 & 6.2 & 9,100 \\
\hline $77 / \mathrm{HR}$ & 1.55 & 232.5 & 9.8 & 11.0 & 1.8 & 9.1 & 13,000 \\
\hline
\end{tabular}
carrier density. The shift causes semiconductor QDs to deviate from the ideal $\alpha_{H}=0$ at the gain peak of an atom, [57] while the broadening modifies the shift effects [58].

To describe $Q D$ dimension and composition variations, we use a Gaussian density distribution:

Table 2: Extracted parameters for the epitaxial QD lasers on silicon with different front facet reflectivity of $\mathrm{C} / \mathrm{HR}, 55 / \mathrm{HR}$ and $77 / \mathrm{HR}$, respectively. 


$$
n_{n, q}^{i n h}=\frac{N_{Q D}^{(2 d)}}{\sqrt{2 \pi} \Delta_{i n h}} \exp \left[-\left(\frac{\hbar\left(\omega_{n, q}^{(0)}-\omega_{n, 0}^{(0)}\right)}{\sqrt{2} \Delta_{\text {inh }}}\right)^{2}\right]
$$

where $N_{Q D}^{(2 d)}$ is the total QD density, $\omega_{n, 0}^{(0)}$ and $\Delta_{i n h}$ are the central transition frequency and standard deviation. Furthermore, we assume carrier populations defined by Fermi-Dirac functions,

$$
\begin{gathered}
f_{n, q}^{\sigma}=\frac{1}{\exp \left[\frac{\left(\varepsilon_{n, q}^{\sigma}-\mu_{\sigma}\right)}{\left(k_{B} T\right)}\right]+1} \\
f_{k}^{\sigma}=\frac{1}{\exp \left[\frac{\left(\varepsilon_{k}^{\sigma}-\mu_{\sigma}\right)}{\left(k_{B} T\right)}\right]+1}
\end{gathered}
$$

where $\varepsilon_{n, q}^{\sigma}$ and $\varepsilon_{k}^{\sigma}$ are the electron and hole (with $\sigma=e$ or $h$ ) energies, $k_{B}$ is Boltzmann constant and $T$ is the active region temperature. Input to the calculations are the total electron and hole densities,

$$
\begin{gathered}
N_{e}=\sum_{n} \sum_{q} n_{n, q}^{i n h} f_{n, q}^{e}+\sum_{k} f_{k}^{e} \\
N_{h}=N_{p}+\sum_{n} \sum_{q} n_{n, q}^{i n h} f_{n, q}^{h}+\sum_{k} f_{k}^{h}
\end{gathered}
$$

which determine the chemical potentials $\mu_{e}$ and $\mu_{h}$. The equations allow for the possibility of p-doping at density $N_{p}$.

The theory is applied to compute gain and $\alpha_{H}$ spectra of an active region consisting of a $7 \mathrm{~nm} \operatorname{In}_{0.15} \mathrm{Ga}_{0.85} \mathrm{As} \mathrm{QW}$ embedding a density of $5 \times 10^{10} \mathrm{~cm}^{-2}$ InAs QDs. The QW is sandwiched between GaAs barriers. In Figure 4(a), each computed gain spectrum shows resonances from one GS and two ESs transitions $n=1,2$, and 3, with degeneracies 1 , 2, and 3, respectively. Also present is a sharp absorption edge at $1.2 \mathrm{eV}$ from the GaAs QW exciton. The calculations are for a relatively uniform QD distribution, with inhomogeneous width $\Delta_{\text {inh }}=10 \mathrm{meV}$, which translates to a spontaneous emission linewidth (full-width at half-maximum) of $\Delta_{s p}=24 \mathrm{meV}$. The conversion is made by solving Eqs.
(2)-(4) and then applying the Kubo-Martin-Schwinger transformation [59]. Figure 4(b) shows the corresponding $\alpha_{H}$ spectra for the GS transition. The dots, indicating the values at gain peak, depict $\alpha_{H}\left(v_{p k}\right)$ changing from negative to positive, which suggests the possibility of lasing at peak gain with $\alpha_{H}=0$.

To explore further, we performed a parametric study involving carrier density and inhomogeneous width. Figure 5(a) shows that for $\Delta_{i n h} \leqslant 16 \mathrm{meV}$, lasing at gain peak with $\alpha_{H}=0$ can occur at certain carrier densities. For $\Delta_{i n h}>16 \mathrm{meV}, \alpha_{H}\left(v_{p k}\right)=0$ is lost, but a minimum still exists. The diamonds in Figure 4(b) indicate the peak gains when lasing with $\alpha_{H}\left(v_{p k}\right)=0$. From a more comprehensive parametric study, we found that the minimum of $\left|\alpha_{H}\left(v_{p k}\right)\right|$ depends on having certain combinations of $\Delta_{i n h}, N_{p}$ and $N_{e}$. To obtain these combinations, one controls the inhomogeneous broadening and p-doping via growth, and determine the desired carrier density by cavity design, using

$$
G_{t h}=G\left(v_{p k}\right)=\frac{1}{\Gamma}\left[\alpha_{a b s}-\frac{1}{2 L} \ln \left(R_{1} R_{2}\right)\right]
$$

where $\Gamma$ is the confinement factor involving the waveguide and the QW embedding the QDs, $L$ the cavity length, $\alpha_{a b s}$ is the intracavity absorption, $R_{1}$ and $R_{2}$ are the facet reflectivities.

We end this section with an explanation of why QD material gain properties computed using a mbt should be viewed differently from those using the more familiar freecarrier theory (fct) $[60,61]$. The free-carrier theory (i.e. ignoring carrier Coulomb interactions) calculates the material gain only from the QDs (black triangles in Figure 6). The modal gain may be written as

$$
G_{\text {mode }}=\Gamma^{f c t} G_{\text {mat }}^{f c t}
$$

where the confinement factor $\Gamma^{f t}$ depends entirely on geometry. Referring to Figure 6, $\Gamma^{\text {fct }}$ may be separated into the overlap of QD and QW volumes and the overlap of QW and laser-mode (WG) volumes:
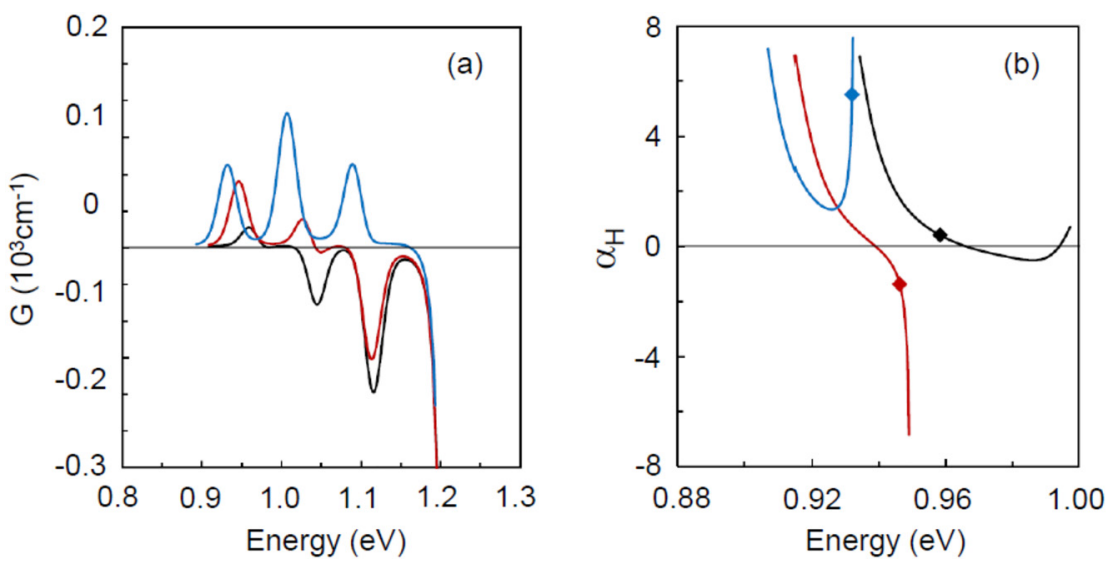

Figure 4: (a) Gain and (b) linewidth enhancement factor $\left(\alpha_{H}\right.$-factor) spectra for InAs QD structure at carrier densities $0.6 \times$, $2 \times, 6 \times 10^{11} \mathrm{~cm}^{-2}$ (black, red, and blue curves, respectively). For the calculations, the $\mathrm{p}$-doped density is $10^{12} \mathrm{~cm}^{-2}$ and inhomogeneous width is $10 \mathrm{meV}$. In Figure 4(b), the photon energy covers only the GS transition, with the dots indicating gain peak values. 


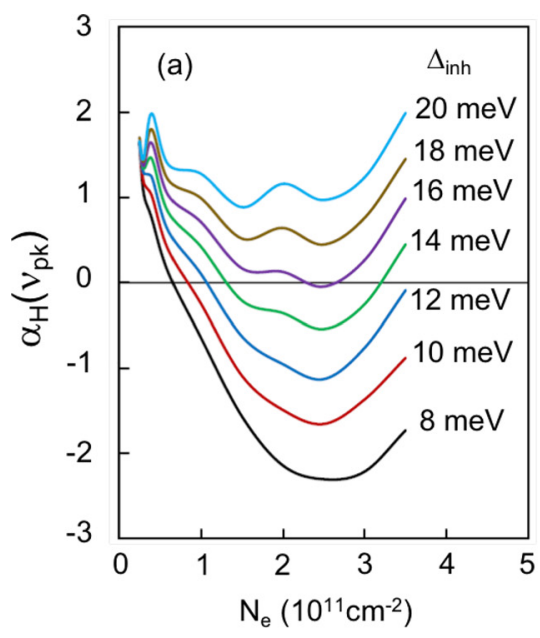

$$
\Gamma^{f c t}=\Gamma_{Q D / Q W} \Gamma_{Q W / W G}
$$

However, even in the free carrier theory, one must still account for scattering resulting in dephasing. The fct does that by introducing a lineshape function with a dephasing rate that is a free parameter.

In the free-carrier calculation, the confinement factor $\Gamma^{f c t} \approx \frac{N_{Q D}^{(2 d)} a h_{q d}}{h_{w g}}$, where $N_{Q D}^{(2 d)}$ is the QD density and $a$ is the QD base area. In the many-body calculation, the confinement factor is $\Gamma^{m b t} \approx \frac{h_{q w}}{h_{w g}}$.

The mbt treats many-body effects, including scattering, more rigorously. To do so, the QW and QDs must be treated as one composite system. [62] Consequently, a material gain that comes only from the QDs no longer makes sense. Instead, the computed material gain is that of

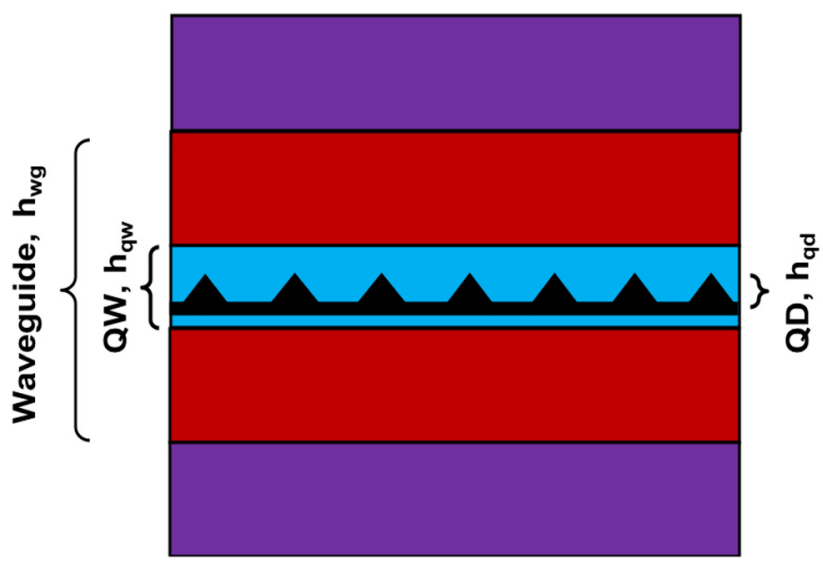

Figure 6: Sketch of QD gain region for illustrating the difference in confinement factor between free-carrier and many-body theories. Between the cladding layers (purple) defining the waveguide are QDs and wetting layer (black) embedded in a QW (blue), which is sandwiched by barriers (red).
Figure 5: (a) Linewidth enhancement factor $\left(\alpha_{H}\right.$-factor) at gain peak and (b) peak gain versus carrier density for ground-state transition, $10^{12} \mathrm{~cm}^{-2} \mathrm{p}$-dope density and inhomogeneous widths as indicated. The diamonds show the peak gains when $\alpha_{H}\left(v_{p k}\right)=0$.

a QW with embedded QDs (blue region in Figure 6). In this case the modal gain is

$$
G_{\text {mode }}=\Gamma^{m b t} G_{m a t}^{m b t}=\Gamma_{Q D / Q W} G_{m a t}^{m b t}
$$

with $G_{m a t}^{m b t}$, one cannot separate out a confinement factor part. The difference between $\Gamma^{f c t}$ and $\Gamma^{m b t}$ is substantial. For a $7 \mathrm{~nm}$ InGaAs QW in a $207 \mathrm{~nm}$ thick waveguide, a back of the envelop estimation gives $\Gamma^{m b t} \approx 0.034$, which is considerably larger than $\Gamma^{f t} \approx 0.0068$, obtained assuming 2.55 monolayers (ML) thick InAs QDs and a fill factor $N_{Q D}^{(2 d)} a$ of $20 \%$. It follows that the many-body material gain is significant smaller than that from the fct. The differences in values have caused some confusion between many-body and free-carrier communities [63]. What is important is the modal gain is the same physical quantity for both theories.

In the experiments, the extraction of the $\alpha_{H}$-factor is performed by using the amplified spontaneous emission (ASE), which relies on direct measurement of the refractive index change and the differential gain as the carrier density is varied by slightly changing the pump current. Since the measurements are performed under continuous waves conditions, the wavelength shift caused by thermal effects must be carefully taken into account. To overcome such an issue, the wavelength red-shift due to thermal effects, measured by varying the pump current right above threshold, is subtracted from the wavelength blueshift measured below threshold. Following this protocol, the fitting error is estimated below $1 \%$, which means that the extraction of the $\alpha_{H}$-factor is accurate. Figure 7 illustrates the measured $\alpha_{H}$-factor at threshold and for the gain peak as a function of power reflectivity of the front facet. The measured values lie within a range from 0.5 (C/HR) to about 0.7 (55/HR) and then close to unity (77/HR). The slight increase of the linewidth enhancement factor is mostly attributed to the decrease of the differential gain 


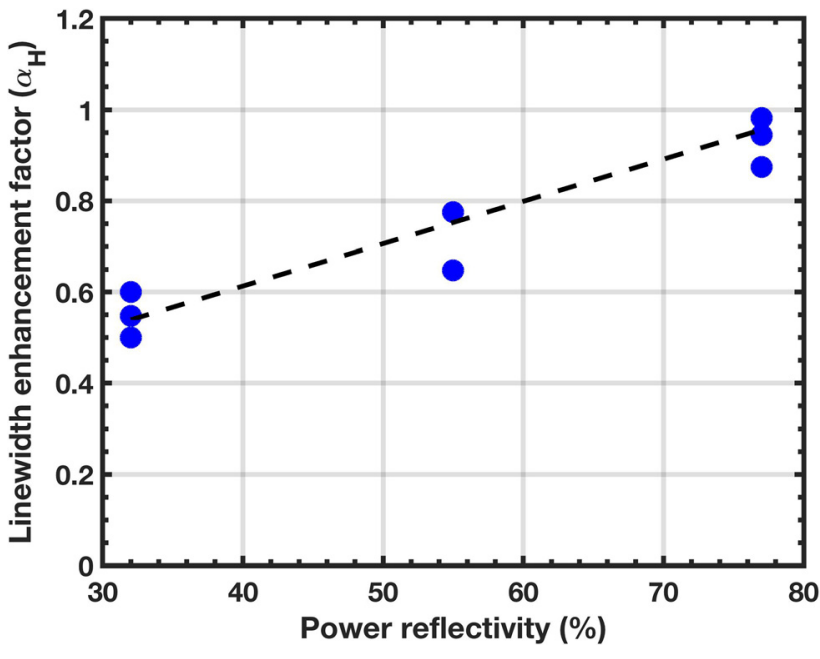

Figure 7: The linewidth enhancement factor ( $\alpha_{H}$-factor) measured as a function of the front facet power reflectivity. The back-facet is HRcoated. The dashed line is a guide to the eyes.

taking place for longer operating wavelengths. Figure 7 proves that near zero $\alpha_{H}$-factors can be obtained with QD lasers on silicon, which are attributed to the reduced size dispersion owing to the narrow inhomegeneous width.

Taking a cavity volume of $9.0 \times 10^{-10} \mathrm{~cm}^{-3}$, a carrier lifetime $\tau_{e}$ of $0.1 \mathrm{~ns}$, and the electron charge of $q=1.6 \times 10^{-19} \mathrm{C}$, the carrier density at threshold $N_{S}$ can be computed from the relationship $N_{s}=\frac{I_{s} \tau_{e}}{q V} N_{Q W} d_{Q W}$ with $N_{Q W}=5$ the number of QWs, and $d_{Q W}=7 \mathrm{~nm}$ the width of the QW (see Figure 2(b)). Depending on the facet coating, $N_{s}$ is found to lie from 0.3 to $0.5 \times 10^{11} \mathrm{~cm}^{-2}$ with respect to the threshold current $I_{s}$ that ranges from 15 to $24 \mathrm{~mA}$. Using those $N_{s}$ values and taking an inhomogeneous width of $10 \mathrm{meV}$, Figure 5(a) indeed well positions the linewidth enhancement factor at the gain peak $(0.96 \mathrm{eV})$ between 0.5 to about one which proves the excellent agreement between the presented measurements and the aforementioned simulations. Let us note that taking into account the spreading of the optical field into the cladding regions, the nominal value of the $\alpha_{H}$ is enhanced by $1+\eta$ with $\eta=G \lambda /\left(2 \pi n_{e}\right)$ with $G$ the material gain per unit length, $\lambda$ the lasing wavelength, and $n_{e}$ the effective index [64]. Assuming a material gain for the QD lasers under study of about a few hundreds of inverse centimeters, one found here that $\eta<1$ and that $\alpha_{H}$ is not impacted by the waveguide structure [4].

\section{External optical feedback (EOF)}

\subsection{Basic theory}

The physical processes involved in a semiconductor laser under EOF are schematically described in Figure 8. The amplitude-phase coupling in the active region between the feedback light field and the intra-cavity one is ruled out by field fluctuations in amplitude $(|\Delta E|)$ and phase $(\Delta \Phi)$. EOF is coupled into the laser cavity through the output facet and causes a perturbation on the photon density. Such a perturbation leads to a fluctuation of the carrier density, and thus the optical gain. The intensity fluctuation is then modulated by the damping effect and linked to the optical gain, where the gain variation impacts the refractive index through the $\alpha_{H}$-factor, hence leading to a deviation of the lasing wavelength. In addition, the phase fluctuation induced from the returned field is also associated to the wavelength fluctuation. The interaction of the intensity and phase loop makes the nonlinear laser's dynamic under EOF very complex with the birth of severe optical instabilities such as CC $[8,65]$.

The complex electric field of a semiconductor laser under EOF can be described as follows [66],

$$
\begin{aligned}
\frac{d E}{d t}= & \left(j\left(\omega-\omega_{0}\right)-\frac{j}{L} \int_{(L)} \frac{\partial W / \partial N}{\partial W / \partial \omega} \Delta N d z\right) E(t) \\
& -\frac{2 C_{k}}{\tau_{\text {in }}} \sqrt{r_{\text {ext }}} E(t-\tau)
\end{aligned}
$$

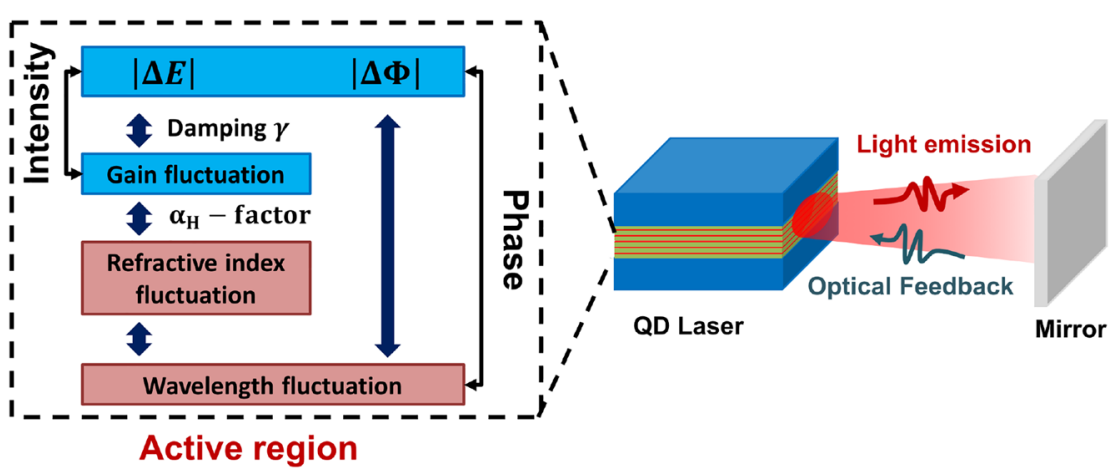

Figure 8: Physical processes involved in a quantum dot (QD) laser with external optical feedback (EOF). 
with $\omega, \omega_{0}$ the lasing frequencies with and without optical feedback, $r_{\text {ext }}$ the EOF strength defined as the ratio between the reflected power and free-space emitting power at the front facet, $\tau$ the external round-trip time, $L$ the laser cavity length, $\tau_{i n}$ the cavity photon round-trip time, and $C_{k}$ the external coupling coefficient of the facet $(k=r, l$ for rear $(r)$ or front (l) respectively) defined by [67]

$$
C_{k}=\frac{j \tau_{\text {in }}}{2}\left(1-r_{k}^{2}\right) \frac{\partial W / \partial r_{k}}{\partial W / \partial \omega}
$$

with $r_{k}$ the amplitude reflectivity, and $W$ the Wronskian operator which stands for a FP laser as [66]

$$
W=2 j \beta r_{r}\left(r_{l} r_{r} e^{-2 j \beta L}-1\right)
$$

where $\beta$ is the complex propagation wavevector. The dependence of the Wronskian on the facet reflectivity is used to take into account EOF coming from a distant reflecting point of amplitude reflectivity $\sqrt{r_{\text {ext }}}$ (in Eq. (14)). Lastly, the dynamic evolution of the carrier density is ruled out by the usual rate equation

$$
\frac{d N}{d t}=\frac{I}{e}-\frac{N}{\tau_{c}}-\frac{N}{\tau_{S R H}}-G|E|^{2}
$$

where $N, \tau_{c}, G$, and $I$ are the carrier density within the active zone, the carrier lifetime, the optical gain, and the injected current, respectively. The lifetime $\tau_{S R H}$ is incorporated into the carrier equation in order to take into account the Shockley-Read-Hall (SRH) non-radiative recombinations that can be induced by the defects and threading dislocations in epitaxial QD lasers on silicon [20]. The effective carrier lifetime is then rewritten such as

$$
\tau_{e}^{-1}=\tau_{c}^{-1}+\tau_{S R H}^{-1}
$$

The reflection sensitivity of the epitaxial QD lasers on silicon is analyzed from the following equations [68]

$$
f(\Delta \omega \tau)=\Delta \omega \tau+X \sin \left(\Delta \omega \tau+\omega_{0} \tau+\tan ^{-1} \alpha_{H}\right)
$$

with

$$
\Delta \omega \tau=\left(\omega-\omega_{0}\right) \tau
$$

and

$$
X=2 C_{k} \frac{\tau}{\tau_{\text {in }}} \sqrt{1+\alpha_{H}^{2}} \sqrt{r_{\text {ext }}}
$$

while the second equation reads as

$$
r_{\text {crit }}=\frac{\tau_{i n}^{2}\left(K f_{\mathrm{R}}^{2}+1 / \tau_{e}\right)^{2}}{16 C_{l}^{2}}\left(\frac{1+\alpha_{H}^{2}}{\alpha_{H}^{4}}\right)
$$

where the damping factor $\gamma=K f_{\mathrm{R}}^{2}+1 / \tau_{e}$ with $K$ a constant refers as the $K$-factor and $f_{R}$ the relaxation oscillation frequency. Together Eqs. (19), (21) and (22) give insights on how to lift-up the reflection insensitivity of any semiconductor laser. The former tells us that depending on the value of the coefficient $X$, the optical feedback induced frequency shift can exhibit two different behaviors: in the low feedback regime $(X<1)$, Eq. (19) has only one solution whereas for $X<1$, Eq. (19) has more than one solution due to the increase of external cavity modes (ECM), in a number increasing with $X$ hence increasing the modal competition and laser instabilities.

As an example, Figure 9 illustrates the number of solutions of $f(\Delta \omega \tau)=0$. Simulations are performed for different values of $X$ assuming either a QW or QD laser with as-cleaved front facet and a $1.3 \mathrm{~mm}$ long cavity length for both cases. In order to better simulate a reflection taking place inside a PIC, the external cavity length is fixed to $10 \mathrm{~cm}$. Three different values of EOF strength $r_{e x t}$ are considered namely $-35 \mathrm{~dB}$ (green), $-25 \mathrm{~dB}$ (red), and $-20 \mathrm{~dB}$ (blue). First, Figure $9 \mathrm{a}$ ) shows the situation for the QW laser with $\alpha_{H}=4$. It is clear that any EOF strength beyond $-30 \mathrm{~dB}(X>1)$ makes the laser no longer stable. This is illustrated from the magnified amplitude of the sine function leading to several intersection points with the $x$ axis. In such a way, several ECMs coexist leading to strong mode competitions visible in the optical spectrum. Reducing the number of ECMs can be achieved either by enhancing the $Q$-factor of the laser cavity $[4,71]$ or by reducing the $\alpha_{H}$-factor, which are exactly what we do with the QD lasers on silicon. As a consequence of that, Figure 9(b) displays the case of the epitaxial QD laser with $\alpha_{H}=1$ (namely taking the largest measured value from Figure 7). Simulations show that even at $-20 \mathrm{~dB}$ EOF strength $(X>1)$, the amplitude of the sine function is much more constrained hence implying that the QD laser exhibits more stability than its QW counterpart with no ECMs excited. As for the angular emitting frequency $\omega$, it depends on the phase $\varphi=\omega_{0} \tau+\tan ^{-1}\left(\alpha_{H}\right)$ occurring in Eq. (19). Here, the phase $\varphi$ is taken in such a way that the sine function intersects at the origin meaning that $\omega \doteq \omega_{0}$. In an actual configuration, $\varphi$ cannot be controlled and the sine function can slightly mover apart from the origin, which means that $\omega$ does not necessarily equal $\omega_{0}$, but can be very close to providing the feedback strength is not too large $(X \sim 1)$. This condition is usually fulfilled in PICs where typical reflections levels remain most likely not beyond $-30 \mathrm{~dB}$. Nevertheless, those simulations qualitatively confirm that the utilization of a QD active media does procure a much higher stability against EOF hence resulting from the lower value of $X$ thanks to the smaller linewidth enhancement factor. 

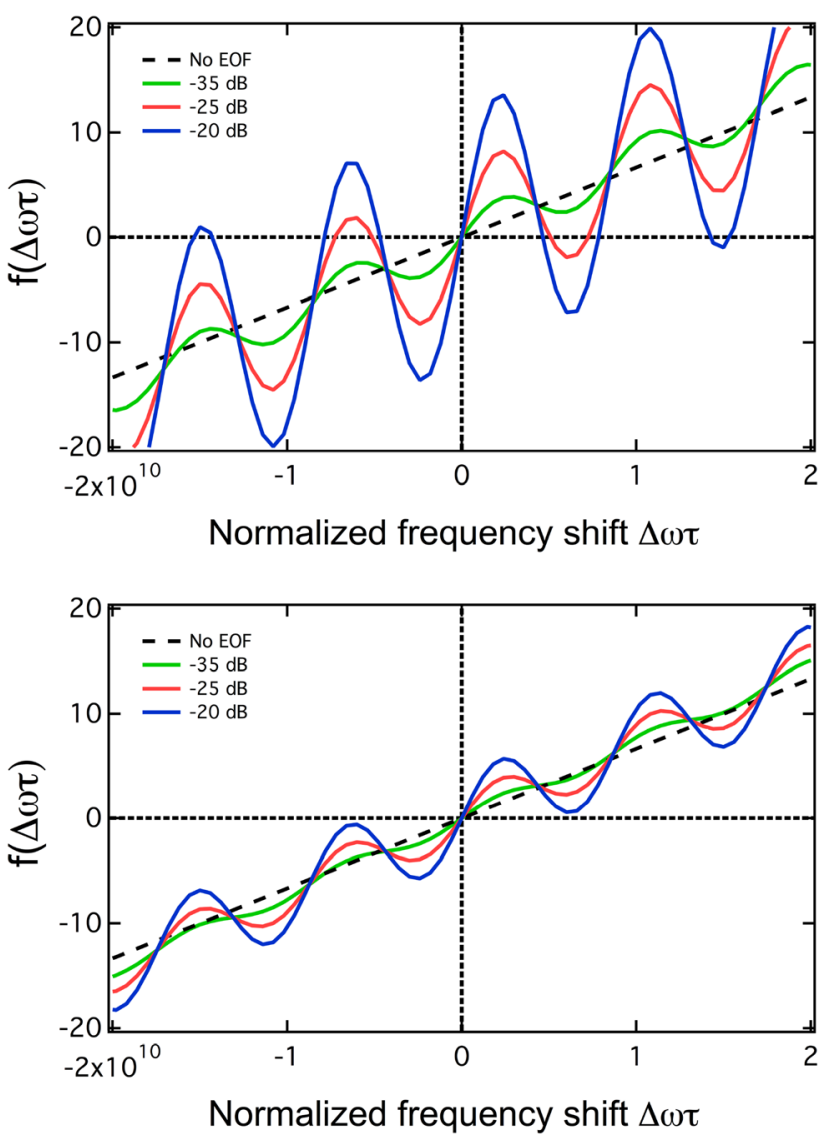

Figure 9: Number of solutions for (a) the QW laser with $\alpha_{H}=4$, and for (b) the epitaxial QD laser with $\alpha_{H}=1$ and. In both cases, the EOF strength $r_{\text {ext }}$ is fixed to $-35 \mathrm{~dB}$ (green), $-25 \mathrm{~dB}$ (red), and $-20 \mathrm{~dB}$ (blue). The black dashed line corresponds to the case without EOF $\left(r_{e x t}=0\right)$. The external cavity length is fixed to $10 \mathrm{~cm}$.

On the other hand, Eq. 22 gives the maximum feedback ratio that can be practically tolerated for a stable laser operation into a communication system in compliance with the IEEE standards. The critical feedback level $r_{\text {crit }}$ corresponds to the minimum feedback strength that needs to be considered in order to observe the undamping of the relaxation oscillations. The exaltation of the relaxation oscillations further degenerates into the $\mathrm{CC}$ that is typically determined at the point where the laser linewidth begins to significantly broaden [12]. When the laser operates within such a regime, the spontaneous emission increases. The noise power $P_{\text {laser }}$ of the laser received at the photodiode can be directly expressed as $P_{\text {laser }}=\left(R_{L} M^{2} S^{2} \Phi^{2} B_{n}\right) \times R I N$ with $R_{L}$ the load resistance, $M$ the avalanche factor, $S$ the optical sensitivity of the photodiode, $\Phi$ the energy flux applied on the photodiode, $B_{n}$ the noise bandwidth and RIN. This equation shows that any increase of the RIN beyond the critical feedback level raises the $P_{\text {laser }}$ and the power penalty for error free operation, which is a severe drawback for high speed applications [10].

\subsection{Directions toward reflection insensitivity}

In order to reduce the coupling of the QD laser to the external world (i.e., the external cavity), several optimization paths can be considered.

(1) First, a higher front facet reflectivity (i. e., a lower $C_{k}$ ) is to be preferred, in order to reduce the coupling towards the external world. In this work, the best optimized devices are left as-cleaved meaning that the power reflectivity of the front facet is $32 \%\left(C_{l}=0.6\right)$, which offers a good balance between a high feedback tolerance and sufficient optical output power. From Eq. (22), the increase of the cavity photon roundtrip time with longer cavities also enhances the feedback resistance without overly affecting the lasing wavelength. Here, we use a $1.35 \mathrm{~mm}$ long cavity, which constitutes a good compromise to cope with high feedback resistance and high laser performance.

(2) Second, from Eq. (22), a large damping factor is beneficial for enhancing reflection insensitivity, which is exactly what happens with the epitaxial QD lasers. Indeed, if the laser dynamics are heavily damped, the gain fluctuation introduced by the photon-density variation can be suppressed. Figure 10 shows the damping factor for the different lasers plotted as a function of the squared relaxation oscillation frequency extracted from the curve-fitting of the RIN (not shown here) [70]. Whatever the power reflectivity applied on the front facet, the damping is always found much higher than that of a classical QW laser [71]. Note that the laser having the larger cavity photon lifetime (77/HR) has the highest damping factor because the gain is reduced.

(3) Third, decoupling the interaction between the intensity and phase is required in order to minimize the $\alpha_{H}$-factor. From Eq. 22, the smaller the $\alpha_{H}$-factor, the weaker the coupling towards the external cavity, the higher the critical feedback level. In QD lasers, the magnitude of the linewidth enhancement factor strongly depends on the QD size dispersion. Thus, it is of paramount importance to reduce such dispersion during the self-organized growth technique of the epitaxy process in order to narrow the inhomogeneous broadening of the gain, which directly transforms into a smaller $\alpha_{H}$-factor as shown in Figure 7. For QW lasers, the linewidth enhancement factor is a more or less constant material parameter ranging from two to about five due to the linear dependence of both the refractive index and gain [71]. The band structure of a QD laser 
contains localized energy levels, which are coupled via relatively slow scattering rates, and thus not occupied through an equal distribution. The usually defined $\alpha_{H}$-factor is therefore non-constant in QD lasers and depends on the operation point. In this work, we show that, thanks to the very narrow inhomogeneous width (10 meV), epitaxial QD lasers on silicon can reach nearzero $\alpha_{H}$ at threshold and exhibit a limited increase beyond which is in favor of high EOF resistance [72].

(4) Fourth, in QD lasers, the stability of the QD laser strongly depends on the GS-ES contrast ratio. A large ratio means that no GS-ES switching dynamics occur when increasing the bias current, which prevents any degradation of the laser's performance and allows a higher degree of stability against EOF [73].

(5) Fifth, in order to increase the feedback resistance, a cavity with a very high $Q$ is to be considered by reducing internal loss and/or mirror loss [74]. Taking into account the $Q$ factor, Eq. 21 can be recast as follows:

$$
X=\omega \tau Q^{-1} \sqrt{1+\alpha_{H}^{2}} \sqrt{r_{e x t}}
$$

Eq. 23 gives insight on how the $Q$ impacts the laser's feedback sensitivity. When the $Q$ is large, the $X$ coefficient can be kept to a very low value $(X<1)$ thus providing a relative feedback insensitivity regardless of the feedback strength [17]. Consequently, it was demonstrated that $r_{\text {crit }} \propto Q^{2} / 1+\alpha_{H}^{2}$ hence showing that the $r_{\text {crit }}$ greatly scales up not only with the $\alpha_{H}$ but also with the $Q$ of the laser cavity [69].

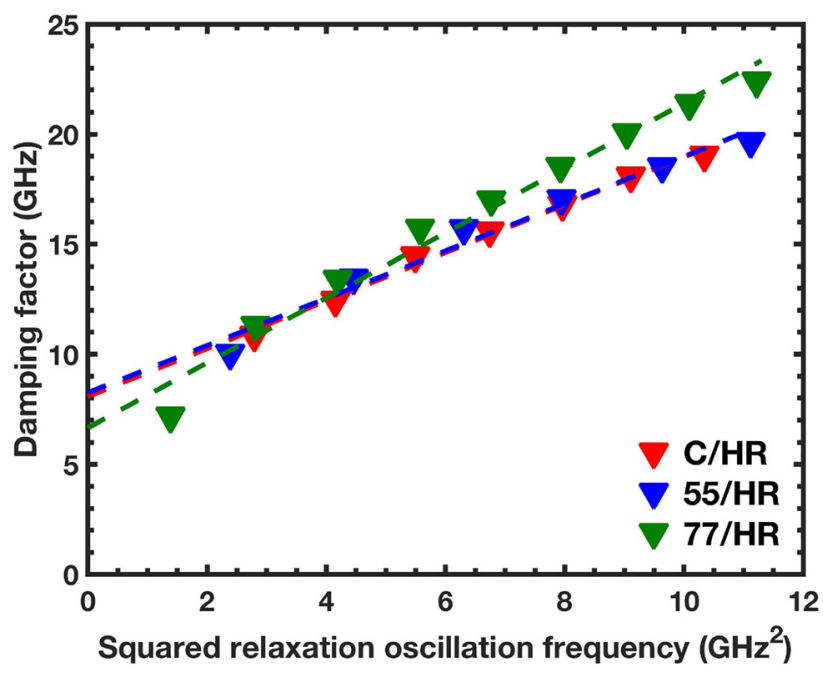

Figure 10: The damping factor $(\gamma)$ as a function of the squared relaxation oscillation frequency $\left(f_{R O}^{2}\right)$ for epiaxial QD lasers on silicon with facets' power reflectivities such as C/HR, 55/HR and 77/ $H R$, respectively.
(6) Finally, the epitaxial defects accelerate SRH recombination lifetime $\tau_{S R H}$, which is inversely proportional to the defect density. A recent paper showed that the SRH recombination plays a role in the degradation of QD lasers on silicon [75]. Figure 11 gives the computed values of the critical feedback level as a function of the $\alpha_{H}$-factor for $\tau_{S R H}$ decreasing from 5 to $0.1 \mathrm{~ns}$. Results show that the critical feedback level is up-shifted by several orders of magnitude, which might be a possible situation occurring in the epitaxial QD lasers on silicon.

(7) Finally, let us stress that the amount of power $P$ of light that is effectively fed-back into the active region remains difficult to estimate. However, it can be approached by calculating the following integral

$$
P=\frac{2}{\pi \theta_{\alpha} \theta_{\beta}} \iint d \alpha d \beta \exp \left[\frac{-2\left(\alpha^{2}+\beta^{2}\right)}{\theta_{\alpha} \theta_{\beta}}\right]
$$

where $\theta_{\alpha} \times \theta_{\beta}$ is the divergence of the laser's beam whereas $\alpha$ and $\beta$ are the tilted angles of the optical fiber. Assuming $\alpha \times \beta=4^{\circ} \times 4^{\circ}$ one can estimate that the amount of light recoupled into the active region is on the order of $-30 \mathrm{~dB}$ against $-40 \mathrm{~dB}$ with $8^{\circ}$. Eq. 24 shows that the smaller the beam divergence, the higher the optical power fed-back into the active region. A compromise has then to be found between beam quality and level of reflection sensitivity that can be tolerated.

In conclusion, based on the different aforementioned elements, researchers can make an informed judgment about which type of design optimizations would be the most suitable for raising the reflection insensitivity.

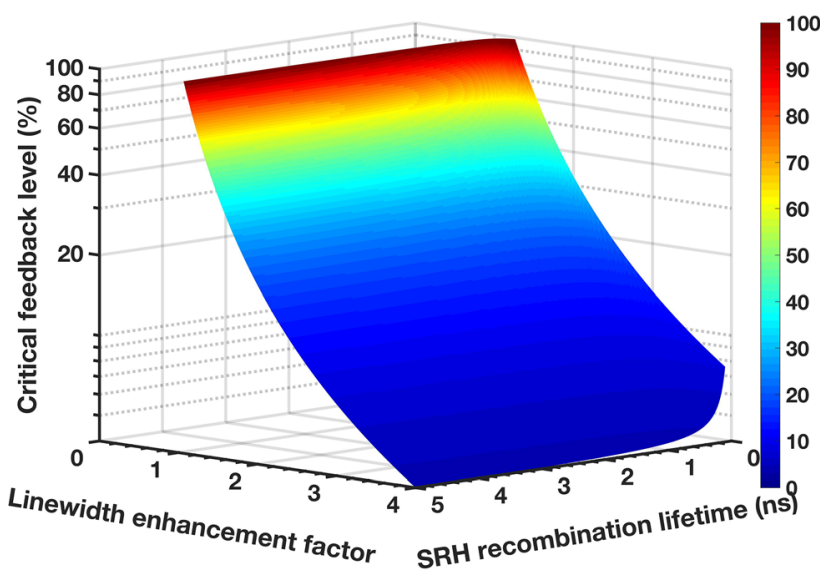

Figure 11: The critical feedback level $\left(r_{\text {crit }}\right)$ as a function of the linewidth enhancement factor $\left(\alpha_{H}\right)$ and SRH recombination lifetime $\left(\tau_{S R H}\right)$. 


\subsection{Experimental results}

Now, we experimentally compare the optical feedback dynamics between an InAs/GaAs QD FP laser directly grown onto silicon and a hybrid QW laser heterogeneously bonded on silicon. The QD laser under consideration is the one with $\mathrm{C} / \mathrm{HR}$ that is to say the laser for which the cleaved front facet $\left(C_{l}=0.6\right)$ is the most coupled to the external cavity. In the experiments, the emission from the QD (QW) laser front facet is coupled with an anti-reflection (AR) coated lens-end fiber, and through the same interface, part of the light is reflected in the fiber back to the laser cavity from a distance of around $7 \mathrm{~m}$, which corresponds to an external cavity frequency of $14 \mathrm{MHz}$. Even though a PIC does not experience such long-delay optical reflections, this configuration is studied because it corresponds to the most stringent feedback conditions for the lasers. Since both lasers are operated within the long-delay configuration hence $f_{R} \gg 1 / \tau$ the impact of the feedback phase is negligible. On the feedback path, $90 \%$ coupled power is directed to the backreflector (BKR) that consists of a mirror and a variable optical attenuator (VOA). The amount of return power is controlled and quantified by the feedback strength $r_{e x t}$, defined as the ratio between the reflected power and free-space emitting power at the front facet. A polarization controller is inserted in the feedback path to compensate the fiber dispersion in the external cavity and to maximize the effects of the optical feedback, namely to have the reflected light in the transverse electric (TE) polarization. In our work, the achievable range of $r_{\text {ext }}$ ranges from $0 \%$ to about $20 \%(-7 \mathrm{~dB})$ for the QD laser and from $0 \%$ to about $13 \%(-9 \mathrm{~dB})$ for the $\mathrm{QW}$ laser. The feedback strength variation depends on the coupling ratio of the laser emission in the fiber. Feedback effects are analyzed in the optical and radio-frequency (RF) domains by using an optical spectrum analyzer (OSA) EXFO Optics and an electrical spectrum analyzer (ESA) Rohde \& Schwarz FSU $20 \mathrm{~Hz}-67 \mathrm{GHz}$.

Figure 12 depicts the spectral evolution of both QW and QD lasers as a function of the increase of the feedback strength. Both lasers are biased at $3 \times I_{s}$ and at $20^{\circ} \mathrm{C}$. As shown in Figure 12, the first row shows the mapping in the optical domain whereas the second is the RF (radio-frequency) one. Figure 12 (a) and (c) show that the QW laser does experience a typical route to chaos through a Hopf bifurcation leading to the CC regime where strong broadening of the FP modes occur. The corresponding critical feedback level is estimated to be at $0.3 \%(-25 \mathrm{~dB})$, which indicates the onset of the undamping of the relaxation oscillations. This critical feedback level is in agreement with our calculations [76] and also similar to prior observations made with QW lasers [77]. In comparison, the epitaxial QD laser on silicon demonstrates a high performance with remarkable stability against optical feedback whatever the feedback strength even at the maximum value of $20 \%$. From the optical domain in Figure12(b), only a slight red shift of the FP modes is observed without any mode instability and spectral broadening. This high stability is also confirmed by the RF spectrum (see Figure 12(d)) where no complex dynamics are observed. As aforementioned discussed, the very high degree of feedback tolerance of the QD gain medium is highly dependent on the inhomogeneous broadening due to nanostructure size variations, but through careful optimization, it is possible to show that even epitaxial lasers on silicon display high performance for isolator-free photonic integration. Such a remarkable feature is explained through the low $\alpha_{H}$-factor, measured of 0.5 at threshold that aligns with aforementioned simulations. This low $\alpha_{H}$-factor results from the narrow full-width at half-maximum of $28 \mathrm{meV}$ (inhomogeneous width $10 \mathrm{meV}$ ) [78] and the absence of higher energy states in the lasing emission process even at high bias. Together these features combined with the large damping are linked to the critical feedback level, and for this reason, one can classify these lasers as reflection insensitive when compared to the state-of-the-art commercial QWs. Let us stress that it was also proved that epitaxial QD lasers on silicon are also highly resistant against incoherent EOF [70] originated from other elements such as semiconductor optical amplifiers. In such case, amplified spontaneous emission (ASE) is the dominant contribution. This additional noise that is not correlated to the laser's output is essentially intensity noise, which gets further coupled to the phase noise through the $\alpha_{H}$ hence affecting the laser's coherence. To illustrate this effect, Figure 13(a) depicts the setup for studying the incoherent EOF. The ASE noise from a booster optical amplifier (BOA) is injected directly into the QD laser through a circulator and the ESA is placed at the output of the circulator to record the RF spectrum. The strength of the ASE noise is controlled by adjusting the bias current of the BOA and quantified using the ratio $\left(r_{B O A}\right)$ between the power from the BOA that has reached the laser facet and the free-space power of the laser. Figure 13(b) compares the RF spectrum of the QD laser in solitary case and under 

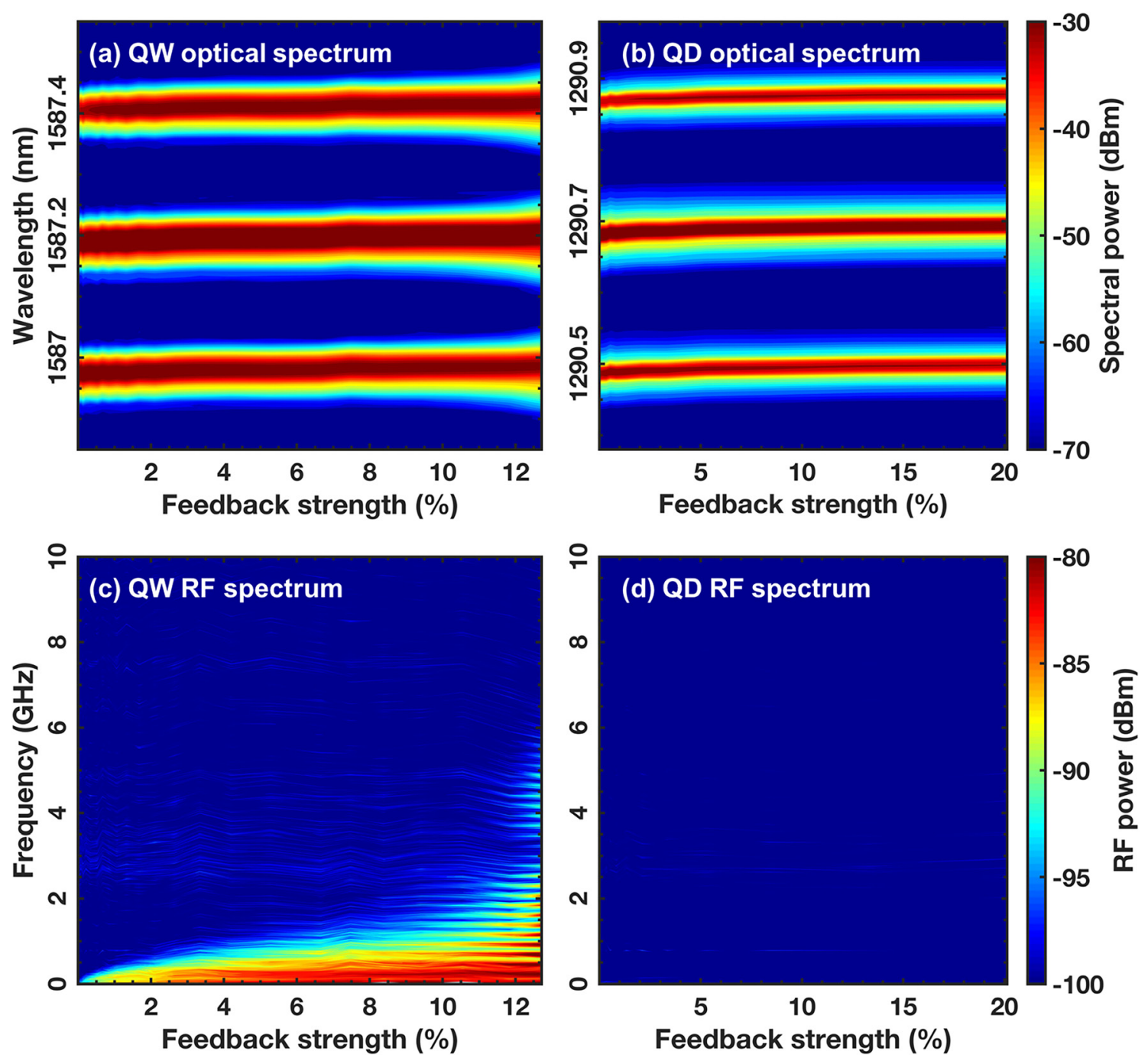

Figure 12: Optical spectrum of (a) QW laser and (b) QD laser, and RF spectrum of (c) QW laser and (d) QD laser. The feedback strength is varied from 0 to $20 \%$ for the QD case (against $13 \%$ for the $\mathrm{QW}$ ).

incoherent optical feedback with $r_{B O A}=86 \%$. As shown, the two spectra are perfectly overlapped, indicating a high tolerance against ASE noise. It is noted that the linewidth broadening due to optical feedback is not observed in the optical domain (not shown) since no complex dynamics occurs in the RF spectrum. Overall, these results prove that epitaxial QD lasers on silicon exhibit a superior resistance to both coherent and incoherent EOF which is a first importance for future coherent communications in PICs. (a)

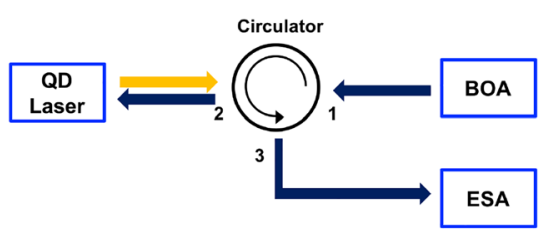

(b)

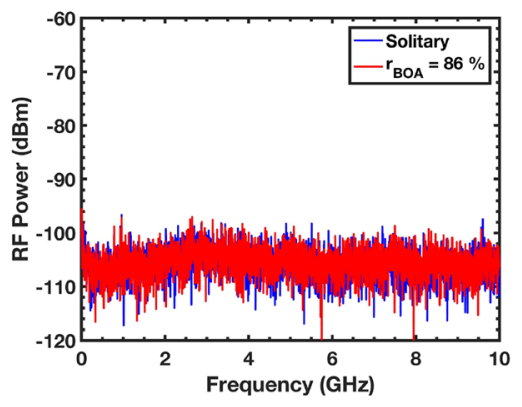

Figure 13: (a) Schematic representation of the incoherent EOF setup. (b) RF spectrum in solitary case and under $86 \%$ incoherent EOF for the epitaxial QD laser on silicon at $3 \times I_{s}$. 


\section{Conclusions}

In this review paper, we shed new insights on reflection insensitive silicon based semiconductor lasers based on QD technology. In particular, we provide a comprehensive study illustrating the potential of such lasers against EOF. To do so, we discuss the connection between the material properties and the ultra-low reflection sensitivity that is achieved. In other words, we link the physical properties of those semiconductor atoms to the laser physics and the large stability observed under both coherent and incoherent optical feedbacks. We also give more details about the crystal growth procedure used to reduce the linewidth enhancement factor through the tight control of the QD size dispersion. We explain that reducing the inhomogeneous width is vital for enhancing the reflection insensitivity. We also show that whatever coating applied on the front facet, all QD lasers under study remain perfectly stable, which is an important result for further optimizing the device performance. We also provide a complete section indicating clear directions toward optical feedback insensitivity. Finally, we perform simulations using a semi-classical laser model including mbt and predicting near zero linewidth enhancement factor in agreement with the experiments. We explain why the many-body approach is more rigorous for QD lasers wherein the QW and QDs are treated as one composite system. In this context, the confinement factor playing a role in the material gain is nicely revisited. Overall, this work on physics and applications of QD lasers provides novel insights for designing high performance reflection insensitive semiconductor lasers, withstanding feedback rates much above the requirement dictated by the IEEE 802.3. These results show the possibility to integrate lasers and other optical components without invoking the need of an optical isolator. Other applications requiring improved coherence and precisely controlled light sources will also benefit from these distinctive attributes and be considered in future developments.

Acknowledgment: Authors acknowledge the financial support of ARPA-E (DE-AR0001042), the Institut Mines Télécom (IMT), the U.S. Department of Energy (DOE) under Contract No. DE-NA0003525. Dr. Chow also thanks the Technical University Berlin (TUB) for hospitality and the German Research Foundation collaborative research center 787 for travel support. This work was performed in part, at the Center of Integrated Nanotechnologies (CINT), an office of Science User Facility operated by the US DOE, Office of Science.
Author contribution: All the authors have accepted responsibility for the entire content of this submitted manuscript and approved submission.

Research funding: None declared.

Employment or leadership: None declared.

Honorarium: None declared.

Conflict of interest statement: The authors declare no conflicts of interest regarding this article.

\section{References}

[1] J. C. Norman, D. Jung, Y. Wan, and J. E. Bowers, “Perspective: The future of quantum dot photonic integrated circuits," $A P L$ Photonics, vol. 3, no. 3, p. 030901, 2018.

[2] D. Liang and J. E. Bowers, "Recent progress in lasers on silicon," Nat. Photon., vol. 4, no. 8, pp. 511-517, 2010.

[3] J. C. Norman, J. Daehwan, Z. Zhang, et al., "A review of highperformance quantum dot lasers on silicon," IEEE J. Quant. Electron., vol. 55, no. 2, pp. 1-11, 2019.

[4] Z. Zhang, D. Jung, J. C. Norman, W. W. Chow, and J. E. Bowers, "Linewidth enhancement factor in InAs/GaAs quantum dot lasers and its implication in isolator-free and narrow linewidth applications," IEEE J. Sel. Top. Quant. Electron., vol. 25, no. 6, p. $1900509,2019$.

[5] G. H. Duan, C. Jany, A. Le Liepvre, et al., “Hybrid III”“V on silicon lasers for photonic integrated circuits on silicon," IEEEJ. Sel. Top. Quant. Electron., vol. 20, no. 4, p. 6100213, 2014.

[6] R. Jones, P. Doussiere, J. B. Driscoll, et al., "Heterogeneously integrated InP/Silicon photonics: Fabricating fully functional transceivers," IEEE Nanotechnol. Mag. (Invited paper), vol. 13, no. 2, pp. 17-26, 2019.

[7] K. Schires, N. Girard, G. Baili, G. H. Duan, S. Gomez, and F. Grillot, "Dynamics of hybrid III-V Silicon semiconductor lasers for integrated photonics," IEEE J. Sel. Top. Quant. Electron., vol. 22, no. 6, pp. 43-49, 2016.

[8] D. M. Kane and K. A. Shore, Unlocking Dynamical Diversity: Optical Feedback Effects on Semiconductor Lasers, Hoboken, New Jersey, John Wiley \& Sons, 2005.

[9] D. Lenstra, B. Verbeek, and A. Den Boef, "Coherence collapse in single-mode semiconductor lasers due to optical feedback," IEEE J. Quant. Electron., vol. 21, no. 6, pp. 674-679, 1985.

[10] F. Grillot, B. Thedrez, O. Gauthier-Lafaye, et al., "Coherence collapse threshold of 1.3 micron semiconductor DFB lasers," IEEE Photon. Technol. Lett., vol. 15, no. 1, pp. 9-11, 2003.

[11] L. Bi, J. Hu, P. Jiang, et al., “On-chip optical isolation in monolithically integrated non-reciprocal optical resonators," Nat. Photon., vol. 5, no. 12, p. 758, 2011.

[12] D. Lenstra, T. T. M. van Schaijk, and K. Willians, “Toward a feedback-insensitive semiconductor laser," IEEE J. Sel. Top. Quant Electron, vol. 25, no. 6, p. 1502113, 2019.

[13] D. Huang, P. Pintus, and J. E. Bowers, “Towards heterogeneous integration of optical isolators and circulators with lasers on silicon," Opt. Mater. Express, vol. 8, no. 9, pp. 2471-2483, 2018.

[14] Y. Zhang, Q. Du, C. Wang, et al., “Monolithic integration of broadband optical isolators for polarization-diverse silicon photonics," Optica, vol. 6, no. 4, pp. 473-478, 2019. 
[15] K. Mizutani, K. Yashiki, M. Kurihara, et al., "Isolator free optical I/ 0 core transmitter by using quantum dot laser," in 2015 IEEE 12th International Conference on Group IV Photonics (GFP), Vancouver, BC, Canada, IEEE, pp. 177-178, 2015.

[16] T. S. Rasmussen, Y. Yu, and J. Mork, "Suppression of coherence collapse in semiconductor Fano lasers," Phys. Rev. Lett., vol. 123 , no. 23, p. 233904, 2019.

[17] S. Gomez, H. Huang, J. Duan, et al., "10 Gbps error-free transmission of a high coherent $\mathrm{Si} / \mathrm{III}-\mathrm{V}$ hybrid distributed feedback laser under strong optical feedback," in 2019 IEEE International Photonics Conference (IPC), USA, IEEE, 2019, p. 978.

[18] V. Brac de la Perriere, H. Benisty, A. Ramdane, and A. Lupu, "Active functional devices using parity-time symmetry optics," in Proc. SPIE, Integrated Optics: Physics and Simulations III, Bellingham, Washington USA, SPIE, 2017, p. 10242C:XX.

[19] L. Columbo, J. Bovington, S. Romero-Garcia, F. Siriani, and M. Gioannini, "Efficient and optical feedback tolerant hybrid laser design for silicon photonics applications," IEEE J. Sel. Top. Quant. Electron., vol. 26, no. 2, p. 8301210, 2020.

[20] J. C. Norman, Quantum Dot Lasers for Silicon Photonics (PhD Thesis), California, UC Santa Barbara, 2018.

[21] A. Liu, J. Peters, X. Huang, et al., "Electrically pumped continuous wave 1.3 micron quantum dot lasers epitaxially grown on on-axis (001) GaP/Si," Opt. Letts., vol. 42, no. 2, pp. 338-341, 2017.

[22] D. Jung, Z. Zhang, J. Norman, et al., "Highly reliable low threshold InAs quantum dot lasers on on-axis (001) Si with $87 \%$ injection efficiency," ACS Photon., vol. 5, no. 3, pp. 1094-1100, 2017.

[23] G. Eisenstein and D. Bimberg, Green Photonics and Electronics, Germany, Springer, 2017.

[24] Y. Arakawa and H. Sakaki, "Multidimensional quantum well laser and temperature dependence of its threshold current," Appl. Phys. Lett., vol. 40, no. 11, pp. 939-941, 1982.

[25] N. Kirstaedter, N. Ledentsov, M. Grundmann, et al., "Low threshold, large $T_{0}$ injection laser emission from InGaAs quantum dots," Electron. Lett., vol. 30, no. 11, pp. 1416-1417, 1994.

[26] R. Mirin A. Gossard, and J. Bowers, "Room temperature lasing from InGaAs quantum dot,” Electron. Lett., vol. 32, no. 18, pp. 1732-1734, 1996.

[27] J. Duan, H. Huang, Z. G. Lu, P. J. Poole, C. Wang, and F. Grillot, "Narrow spectral linewidth in InAs/InP quantum dot distributed feedback lasers," App. Phys. Lett., vol. 112, no. 12, p. 121102, 2018.

[28] J. Kwoen, B. Jang, J. Lee, T. Kageyama, K. Watanabe, and Y. Arakawa, "All mbe grown InAs/GaAs quantum dot lasers on onaxis Si (001)," Opt. Express, vol. 26, no. 9, pp. 11568-11576, 2018.

[29] D. O’Brien, S. P. Hegarty, G. Huyet, et al., "Feedback sensitivity of 1.3 micron InAs/GaAs quantum dot lasers," Electron. Lett., vol. 39, no. 25, p. 1819, 2003.

[30] C. Otto, K. Lüdge, and E. Schöll, "Modeling quantum dot lasers with optical feedback: sensitivity of bifurcation scenarios," Phys. Status Solidi B, vol. 247, no.4, pp. 829-845, 2010.

[31] E. A. Viktorov, P. Mandel, I. O'Driscoll, et al., "Low-frequency fluctuations in two-state quantum dot lasers," Opt. Letts., vol. 31, no.15, p. 2302, 2006.

[32] H. Lin, Y. Hong, S. Ourari, T. Huang, and C. Yang, "Quantum dot lasers subject to polarization-rotated optical feedback," IEEE J. Quan. Electron., vol. 56, no.1, p. 2000308, 2020.
[33] M. Dillane, J. Robertson, D. Goulding, A. Hurtado, and B. Kelleher, "Type II excitability with quantum dot lasers: Canards, bistabilities and more," in 2019 Conference on Lasers and Electro-Optics Europe European Quantum Electronics Conference (CLEO/Europe-EQEC), Munich, Germany, Optical Society of America, 2019, p. 978.

[34] H. Su, L. Zhang, A. L. Gray, et al., "High external feedback resistance of laterally loss coupled distributed feedback quantum dot semiconductor lasers," IEEE Photon. Technol. Letts., vol. 15, , no.11, p. 1504, 2003.

[35] S. Azouigui, B. Dagens, F. Lelarge, et al., "Optical feedback tolerance of quantum-dot - and quantum-dash-based semiconductor lasers operating at $1.55 \mu \mathrm{m}$," IEEE J. Sel. Top. Quant. Electron., vol. 15, no. 13, p. 764, 2009.

[36] M. Matsuda, N. Yasuoka, K. Nishi, et al., "Low-noise characteristics on $1.3 \mu \mathrm{m}$ wavelength quantum-dot DFB lasers under external optical feedback," In 2018 IEEE International Semiconductor Laser Conference (ISLC), Santa Fe, NM, USA, IEEE, p. 978, 2018.

[37] H. Huang, L. C. Lin, C. Y. Chen, et al., “Multimode optical feedback dynamics in InAs/GaAs quantum dot lasers emitting exclusively on ground or excited states: transition from short- to long-delay regimes," Opt. Express, vol. 26, no. 2, pp. 1743-1751, 2018.

[38] K. Lüdge, Nonlinear Laser Dynamics: From Quantum Dots to Cryptography, New Jersey, Wiley, 2012.

[39] F. I. Zubov, M. V. Maximov, E. I. Moiseev, et al., "Observation of zero linewidth enhancement factor at excited state band in quantum dot laser," Electron. Lett., vol. 51, no. 21, pp. 16861688, 2015.

[40] L. C. Lin, C. Y. Chen, H. Huang, et al., "Comparison of optical feedback dynamics of InAs/GaAs quantum-dot lasers emitting solely on ground or excited states," Opt. Lett., vol. 43, no. 2, pp. 210-213, 2018.

[41] A. Y. Liu and J. E. Bowers, "Photonic integration with epitaxial III”“V on silicon,” IEEEJ. Sel. Top. Quant. Electron., vol. 24, no. 6, pp. 1-12, 2018.

[42] W. W. Chow, S. W. Koch, and M. Sargent, Semiconductor Laser Physics, Germany, Springer, 2011.

[43] J. Wu and P. Jin. "Self-assembly of InAs quantum dots on GaAs (001) by molecular beam epitaxy," Front. Phys. China, vol. 10, no. 1, pp. 7-58, 2015.

[44] V. Shchukin, N. N. Ledentsov, and D. Bimberg, Epitaxy of Nanostructures, Germany, Springer Science Business Media, 2013.

[45] K. Nishi, K. Takemasa, M. Sugawara, and Y. Arakawa. "Development of quantum dot lasers for data-com and silicon photonics applications," IEEEJ. Sel. Top. Quantum Electron., vol. 23, no. 1, pp. 1-7, 2017.

[46] L. Chu, M. Arzberger, G. Böhm, and G. Abstreiter. "Influence of growth conditions on the photoluminescence of self-assembled InAs/GaAs quantum dots," J. Appl. Phys., vol. 85, no. 4, pp. 2355-2362, 1999.

[47] S. Ozdemir, Y. N. Suyolcu, S. Turan, and A. Bulent. "Influence of the growth conditions on the optical and structural properties of self-assembled InAs/GaAs quantum dots for low As/In ratio," Appl. Surf. Sci., vol. 392, pp. 817-825, 2017.

[48] I. Mukhametzhanov, Z. Wei, R. Heitz, and A. Madhukar. "Punctuated island growth: An approach to examination and control of quantum dot density, size, and shape evolution," Appl. Phys. Lett., vol. 75, no. 1, pp. 85-87, 1999. 
[49] Z. Wasilewski, S. Fafard, and J. McCaffrey. "Size and shape engineering of vertically stacked self-assembled quantum dots," J Cryst Growth, vol. 201, pp. 1131-1135, 1999.

[50] J. D. Jackson, Classical Electrodynamics, 3rd ed. New York, NY, Wiley, 1999.

[51] H. Haus and C. Shank, "Antisymmetric taper of distributed feedback lasers," IEEE J. Quant. Electron., vol. 12, no. 9, pp. 532539, 1976.

[52] C. Henry, "Theory of linewidth of semiconductor lasers," IEEE J. Quant. Electron., vol. 18, no. 2, pp. 259-264, 1982.

[53] K. Vahala and A. Yariv, "Semiclassical theory of noise in semiconductor lasers - part II," IEEE J. Quant. Electron., vol. 19, no. 6, pp. 1102-1109, 1983.

[54] W. W. Chow and F. Jahnke, "On the physics of semiconductor quantum dots for applications in lasers and quantum optics," Progr. Quant. Electron., vol. 37, no. 3, pp. 109-184, 2013.

[55] H. C. Schneider, W. W. Chow, and S. W. Koch, "Excitationinduced dephasing in semiconductor quantum dots," Phys. Rev. $B$, vol. 70, no. 23, 2004, https://doi.org/10.1103/PhysRevB.70. 235308, 235308-1-235308-4.

[56] J. Seebeck, T. R. Nielsen P. Gartner, and F. Jahnke, "Polarons in semiconductor quantum dotsand their role in the quantum kinetics of carrier relaxation," Phys. Rev. B, vol. 71, no. 12, 2005, https://doi. org/10.1103/PhysRevB.71.125327, 125327-1-125327-6.

[57] H. C. Schneider, S. W. Koch, and W. W. Chow, "Anomalous carrier-induced dispersion in quantum-dot active media," Phys. Rev. B, vol. 66, no. 4, p. 04130, 2002.

[58] M. Lorke, F. Jahnke, and W. W. Chow, "Excitation dependences of gain and carrier-induced refractive index change in quantum-dot lasers," Appl. Phys. Lett., vol. 90, no. 5, pp. 051112-051114, 2007.

[59] C. H. Henry, R. A. Logan, and F. R. Merritt, "Measurement of gain and absorption spectra in AIGaAs buried heterostructure lasers," J. Appl. Phys., vol. 51, no. 6, pp. 3042-3051, 1980.

[60] M. Asada, Y. Miyamoto, and Y. Suematsu, "Gain and the threshold of three-dimensional quantum-box lasers," IEEE J. Quant. Electron., vol. 22, no. 9, pp. 1915-1921, 1986.

[61] M. Grundmann and D. Bimberg, "Gain and threshold of quantum dot lasers: theory and comparison with experiments," Jpn. J. Appl. Phys., vol. 36, no. 6B, pp. 4181-4187, 1997.

[62] H. C. Schneider, W. W. Chow, and S. W. Koch, "Many-body effects in the gain spectra of highly excited quantum-dot lasers," Phys. Rev. B, vol. 64, no. 11, pp. 115315-115321, 2001.

[63] N. Kirstaedter, O. G. Schmidt, N. N. Ledenstov, et al., "Gain and differential gain of single layer InAs/GaAs quantum dot injection lasers," Phys. Rev. B, vol. 69, no. 9, pp. 1226-1228, 1996.

[64] M. Osinski and J. Buus, "Linewidth broadening factor in semiconductor lasers "“-An overview," IEEE J. Quant. Electron., vol. 23, no. 1, pp. 9-29, 1987.
[65] J. Ohtsubo, "Semiconductor lasers: Stability, instability and chaos," in Springer Series in Optical Sciences, New York, Springer Berlin Heidelberg, 2010.

[66] F. Grillot, G. H. Duan, and B. Thedrez, "Feedback sensitivity and coherence collapse threshold of semiconductor DFB lasers with complex structures," IEEEJ. of Quant. Electron., vol. 40, no. 3, pp. 1-11, 2004.

[67] F. Grillot, B. Dagens, J. G. Provost, H. Su, and L. F. Lester, "Gain compression and above-threshold linewidth enhancement factor in 1.3 micron InAs/GaAs quantum-dot lasers," IEEE J. Quant. Electron., vol. 44, no. 10, pp. 946-951, 2008.

[68] J. Helms and K. Petermann, "A simple analytic expression for the stable operation range of laser diodes with optical feedback," IEEE J. Quantum Electron., vol. 26, no. 5, pp. 833-836, 1990.

[69] S Gomez, H Huang, J Duan, et al., "High coherence collapse of a hybrid III-V/Si semiconductor laser with a large quality factor," IOPJ. Phys. Photon., vol. 26, no. 5, p. 025005, 2020, https://doi. org/10.1088/2515-7647/ab6a74, page Accepted.

[70] H. Huang, Duan J, Dong B, et al., "Epitaxial quantum dot lasers on silicon with high thermal stability and strong resistance to optical feedback," APL Photon., vol. 5, no. 1, p. 016103, 2020.

[71] L. A. Coldren and S. W. Corzine, "Diode lasers and photonic integrated circuits," in Wiley Series in Microwave and Optical Engineering, New York, Wiley, 1995.

[72] J. Duan, H. Huang, D. Jung, et al., "Semiconductor quantum dot lasers epitaxially grown on silicon with low linewidth enhancement factor," App. Phys. Lett., vol. 112, no. 25, p. 251111, 2018.

[73] H. Huang, J. Duan, D. Jung, et al., "Analysis of the optical feedback dynamics in InAs/GaAs quantum dot lasers directly grown on silicon," J. Opt. Soc. Am. B, vol. 35, no. 11, pp. 2780-2787, 2018.

[74] Z. Zhang, H. Wang, N. Satyan, G. Rakuljic, C. T. Santis, and A. Yariv, "Coherent and incoherent optical feedback sensitivity of high-coherence Si/III-V hybrid lasers," in Optical Fiber Communication Conference, San Diego, California, United States, Optical Society of America, 2019, vol. 26, W4E.3.

[75] M. Buffolo, F. Samparisi, L. Rovere, et al., "Investigation of current-driven degradation of 1.3 micron quantum-dot lasers epitaxially grown on silicon," IEEE J. Sel. Top. Quant. Electron., vol. 26, no. 2, pp. 1-8, 2019.

[76] J. Duan, H. Heming, B. Dong, et al., "Dynamic and nonlinear properties of epitaxial quantum dot lasers on silicon for isolatorfree integration," Photon. Res., vol. 7, no. 11, pp. 1222-1228, 2019.

[77] F. Grillot, B. Thedrez, J. Py, O. Gauthier-Lafaye, V. Voiriot, and J. L. Lafragette, "2.5 Gbps transmission characteristics of 1.3 micron DFB lasers with external optical feedback," IEEE Photon. Technol. Letts., vol. 14, no. 1, pp. 101-103, 2002.

[78] W. W. Chow, Z. Zhang, J. C. Norman, S. Liu, and J. E. Bowers, “On quantum-dot lasing at gain peak with linewidth enhancement factor $\alpha_{H}=0$," APL Photonics, vol. 5, no. 2, p. 026101, 2020. 